\title{
DEVELOPMENT OF A NEW FATIGUE DAMAGE MODEL FOR QUARTER-MODAL SPECTRA IN FREQUENCY DOMAIN
}

\author{
UDC 629.5.015.4
}

Original scientific paper

\begin{abstract}
Summary
Floating marine structures are exposed to various environmental loads. When the response of structures caused by these loads can be expressed as a multi-modal spectrum in the frequency domain. To estimate the fatigue damage by these loads, various fatigue damage models have been suggested by many previous researches. The offshore riser system, for example, that is a typical marine structure shows a quarter-modal response. However, existing fatigue damage models are suitable for spectrum with tri-modal in maximum. Consequently, it is necessary to develop a new fatigue damage model that can accurately calculate fatigue damage of quarter-modal spectrum. In this research, the Benasciutti and Tovo model was adopted for developing a new fatigue damage model. And 900 quarter-modal spectra are used to derive a new weighting factor in the Benasciutti and Tovo model by investigating the relationship between the linear weighting factor and the bandwidth parameters. Finally, a new linear weighting factor is derived using the least square method. The suitability of the new fatigue damage model with the derived weighting factor is evaluated by frequency domain and time domain results and their comparison with existing model's results.
\end{abstract}

Key words: $\quad$ Quarter-modal spectrum; Wide-band loading; Fatigue damage model; Rainflow counting method

\section{Introduction}

Floating marine structures are exposed to dynamic loads such as wave, wind, and current. The combination of these loadings can induce more fatigue damage on the structures, since it increases stress range more than that of the individual one. The stress or tension of structures caused by these loads can be expressed as a multi-modal spectrum in the frequency domain. The complex loads are separated or considered at the same time to calculate fatigue damage of the structures.

Fatigue damage calculation method can be considered in either time domain or frequency domain. In time domain, the fatigue damage is calculated with the rainflow counting method [1] which considers stress range and the number of cycles, the S-N diagram characteristics, and the linear cumulative method [2]. It is more accurate than the frequency 
domain fatigue analysis, but this demands more time and cost. On the other hand, the frequency domain method calculates an expected value of fatigue damage by putting the material constant, slope of the S-N diagram, and the spectral moment of response spectrum into a fatigue damage model. The fatigue damage model is an equation derived with the spectral moment and characteristics of the $\mathrm{S}-\mathrm{N}$ diagram as variables in order to approximate the distribution of stress range which is counted using rainflow counting method in time domain methods. The frequency domain method allows a faster calculation than the time domain fatigue analysis, but an accurate result cannot be obtained if the fatigue damage model applied is not appropriate for the target system.

Fatigue damage models can be classified according to development methods. The first one is developing the new probability density function of the stress range calculated by the rainflow counting method. Distribution of the stress range is approximated from a combination of multiple functions. Representative fatigue damage models which have been developed for a wide-band spectrum by this method are the Dirlik model [3] and the Zhao and Baker model [4]. As another alternative, the stress range distribution of the wide-band process is derived by multiplying the correction factor which is expressed by the spectral parameters to Rayleigh stress range distribution. This implies that the wide-band process fatigue damage can be calculated the multiplying the correct factor to the expected fatigue damage of a narrow-band spectrum [5]. The typical models based on this method include the Wirsching and Light model [6] and the Benasciutti and Tovo model [7].

Also, fatigue damage can be classified according to the number of peaks considered in the model. To calculate the fatigue damage of the bi-modal spectrum, the $\mathrm{Fu}$ and Cebon model [8], the Sakai and Okamura model [9], and the Jiao and Moan model [10] were proposed. In addition, there are the fatigue damage models for the tri-modal spectrum such as the Gao and Moan model [11] and the Park et al. model [12].

The typical offshore structure in which its response expressed as a quarter-modal spectrum is a riser, and it is not easy to evaluate fatigue damage considering all kinds of loads that make up the quarter modal spectra at once. Because of the uncertainty of the Vortex Induced Vibration (VIV) which is a vibration of riser induced current load, it is difficult to simulate the coupled analysis of riser considering wave load and current load. Therefore, estimating each fatigue damage from wave load and current load separately is a widely used approach [13]. Some fatigue damage models had been developed for calculating the wave or current induced fatigue damage of offshore structures and investigated [4,10-12,14]. Also, there are some researches try to evaluate fatigue damage of riser with artificial neural networks concept $[15,16]$. However, as a new concept of the mathematical model which can describe the VIV in the time domain is developed recently [17], it is possible to calculate the fatigue damage of riser considering all loads. Consequently, it is necessary to develop a new fatigue damage model that can accurately calculate fatigue damage of the quarter-modal spectrum. In this study, the stress response characteristic of the riser in the FPSO (Floating Production Storage and Offloading) is selected as a typical quarter-modal spectrum. Prior to deriving a new fatigue damage model, the existing wide-band fatigue damage models are reviewed. And the procedure of deriving the new weighting factor in the Benasciutti-Tovo's model is introduced. The fatigue damage model with a new weighting factor is validated by comparing it with the existing wideband fatigue damage models.

\section{Overview of the wide-band fatigue damage models}

The time series process generated from a spectrum should be assumed to be an ergodic stationary Gaussian random process. If the assumption is valid, the generated time series have 
the same statistical characteristic as frequency domain. The characteristic values in the frequency domain and the wide-band fatigue damage models using these values as follows.

\subsection{Spectral moment and bandwidth variable}

The $n^{\text {th }}$ spectral moment is an integral value over frequency of the spectral density function multiplied by the $n^{\text {th }}$ power of the frequency. It is expressed in circular frequency as show in equation (1).

$$
m_{n}=\int_{0}^{\infty} \omega^{n} d \omega
$$

where $\omega$ is a circular frequency, $m_{n}$ is a $n^{\text {th }}$ spectral moment and $S(\omega)$ is a spectral density of $\omega$.

Moreover, the bandwidth variable, which is a function of the spectral moment, is used to identify the shape of a given spectrum and the geometrical characteristic of the frequency distribution, and the most widely used types $\alpha_{1}$ and $\alpha_{2}$ are defined as in equation (2) \& (3).

$$
\begin{aligned}
& \alpha_{1}=\frac{m_{1}}{\sqrt{m_{0} m_{2}}} \\
& \alpha_{2}=\frac{m_{2}}{\sqrt{m_{0} m_{4}}}
\end{aligned}
$$

The $\alpha_{1}$ and $\alpha_{2}$ is between 0 and 1 . As their value becomes closer to 1 , the spectrum is more similar to a narrow-band spectrum. On the other hand, if their value is closer to 0 , the spectrum is closer to wide-band spectrum. In a Gaussian process, peak frequency, $v_{p}$, which is the frequency of peak per unit time, and zero up-crossing frequency, $v_{0}{ }^{+}$, which is the frequency of up-crossing per unit time, are defined as in the following equation (4) \& (5).

$$
\begin{aligned}
& v_{p}=\frac{1}{2 \pi} \sqrt{\frac{m_{4}}{m_{0}}} \\
& v_{0}^{+}=\frac{1}{2 \pi} \sqrt{\frac{m_{2}}{m_{0}}}
\end{aligned}
$$

\subsection{Dirlik model}

This method is a kind of the PDF combination method and has been used in a wideband process fatigue analysis. It was derived by combining one exponential function and two Rayleigh probability distribution functions [3]. A numerical simulation with the main variables $\alpha_{1}$ and $\alpha_{2}$ was performed and the results were fitted to derive the probability density function of a stress range distribution. The expected fatigue damage calculated by the Dirlik model is as shown in equation (6), $k$ is the slope of the $\mathrm{S}-\mathrm{N}$ diagram and $C$ is a material constant [18]

$$
E\left(D_{R F C}\right)=\frac{T_{d} v_{p}}{C}\left(2 \sqrt{m_{0}}\right)^{k}\left[D_{1} Q^{k} \Gamma(1+k)+(\sqrt{2})^{k} \Gamma\left(1+\frac{k}{2}\right)\left(D_{2}|R|^{k}+D_{3}\right)\right]
$$


where coefficients are defined as in equations (7) to (12), and $T_{d}$ is the duration.

$$
\begin{aligned}
& x_{m}=\frac{m_{1}}{m_{0}}\left(\frac{m_{2}}{m_{4}}\right)=\alpha_{1} \alpha_{2} \\
& D_{1}=\frac{2\left(x_{m}-\alpha_{2}^{2}\right)}{1+\alpha_{2}^{2}} \\
& D_{2}=\frac{1-\alpha_{2}-D_{1}+D_{1}^{2}}{1-R} \\
& D_{3}=1-D_{1}-D_{2} \\
& Q=\frac{1.25\left(\alpha_{2}-D_{3}-R^{*} D_{2}\right)}{D_{1}} \\
& D_{3}=1-D_{1}-D_{2}
\end{aligned}
$$

\subsection{Benasciutti and Tovo model}

This model was derived by considering that the expected value $E\left(D_{R F C}\right)$ of the fatigue damage calculated by the rainflow counting method is in a range between $E\left(D_{R C}\right)$ of equation (13), which is determined by range counting method [19], and $E\left(D_{N B}\right)$ of equation (14), which is expected fatigue damage for narrow-band spectrum [7]. The relationship between the three kinds of expected fatigue damage is as shown in equation (15), and the expected the fatigue damage is determined by applying the linear weighting factor of equation (16) to the upper and lower limits of the expected fatigue damage.

$$
\begin{aligned}
& E\left(D_{R C}\right) \cong \frac{v_{p}}{C}\left(2 \sqrt{2 m_{0}} \alpha_{2}\right)^{k} \Gamma\left(1+\frac{k}{2}\right)=E\left(D_{N B}\right) \alpha_{2}^{k-1} \\
& E\left(D_{N B}\right)=\frac{v_{0}^{+}}{C}\left(2 \sqrt{2 m_{0}}\right)^{k} \Gamma\left(1+\frac{k}{2}\right) \\
& E\left(D_{R C}\right) \leq E\left(D_{R F C}\right) \leq E\left(D_{N B}\right) \\
& b_{a p p}=\frac{\left(\alpha_{1}-\alpha_{2}\right)\left[1.112\left(1+\alpha_{1} \alpha_{2}-\left(\alpha_{1}+\alpha_{2}\right)\right) e^{2.11 \alpha_{2}}+\left(\alpha_{1}-\alpha_{2}\right)\right]}{\left(\alpha_{2}-1\right)^{2}}
\end{aligned}
$$

The expected the fatigue damage derived from the relation between $E\left(D_{R C}\right)$ and $E\left(D_{N B}\right)$ is as shown in equation (17).

$$
E\left(D_{R F C}\right)=b_{a p p} E\left(D_{N B}\right)+\left(1-b_{a p p}\right) E\left(D_{R C}\right)=\left[b_{a p p}+\left(1-b_{a p p}\right) \alpha_{2}^{k-1}\right] E\left(D_{N B}\right)
$$




\section{Development of a new fatigue damage model}

\subsection{Method for developing the model}

In order to derive a new fatigue damage model, the Benasciutti and Tovo model was selected. Although this model is not appropriate for evaluating the fatigue of a wide-band quarter-modal spectrum, the procedure of deriving a linear weighting factor was adequate for this study. When the Benasciutti and Tovo model was derived from a numerical simulation of various continuous spectra, the new model also needs to be derived from a wide-band quartermodal spectrum consisting of narrow-band mode peaks. Consequently, it is estimated that the trend of the linear weighting factor according to the spectral variables in the new model will be different from those in the existing models. In this study, the relationship between the linear weighting factors $\alpha_{1}$ and $\alpha_{2}$ was newly defined by a numerical simulation of various wide-band quarter-modal spectra. Fig. 1 shows the detailed model development process of using spectra to derive the linear weighting factor.

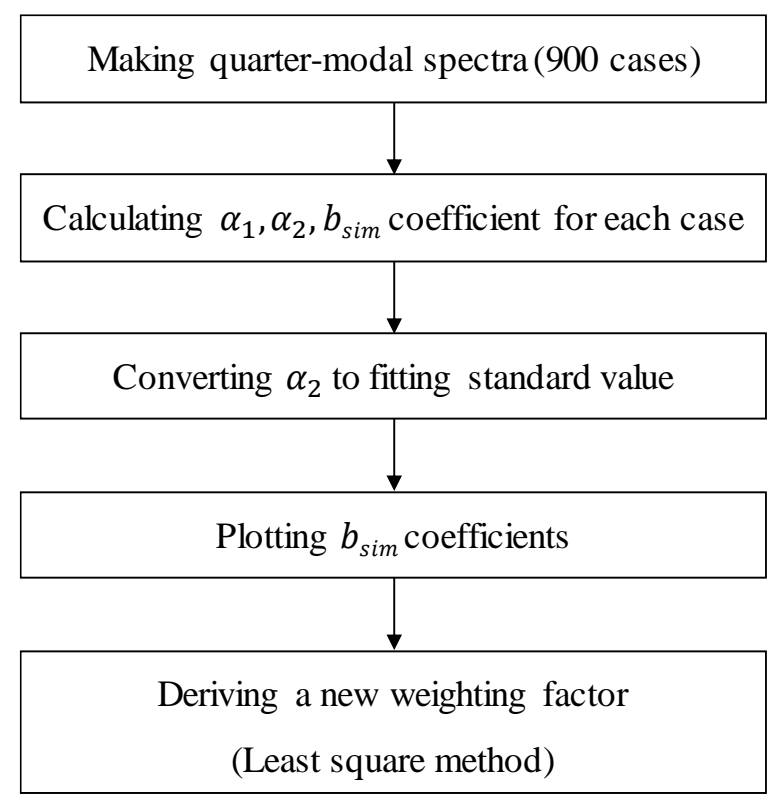

Fig. 1 Model development procedure of new weighting factor

\subsection{Idealized wide-band quarter-modal spectrum}

As shown in Fig. 2, the stress spectrum of the riser in FPSO used in this study consists of four narrow-band mode peaks and every peak was idealized as rectangles. 


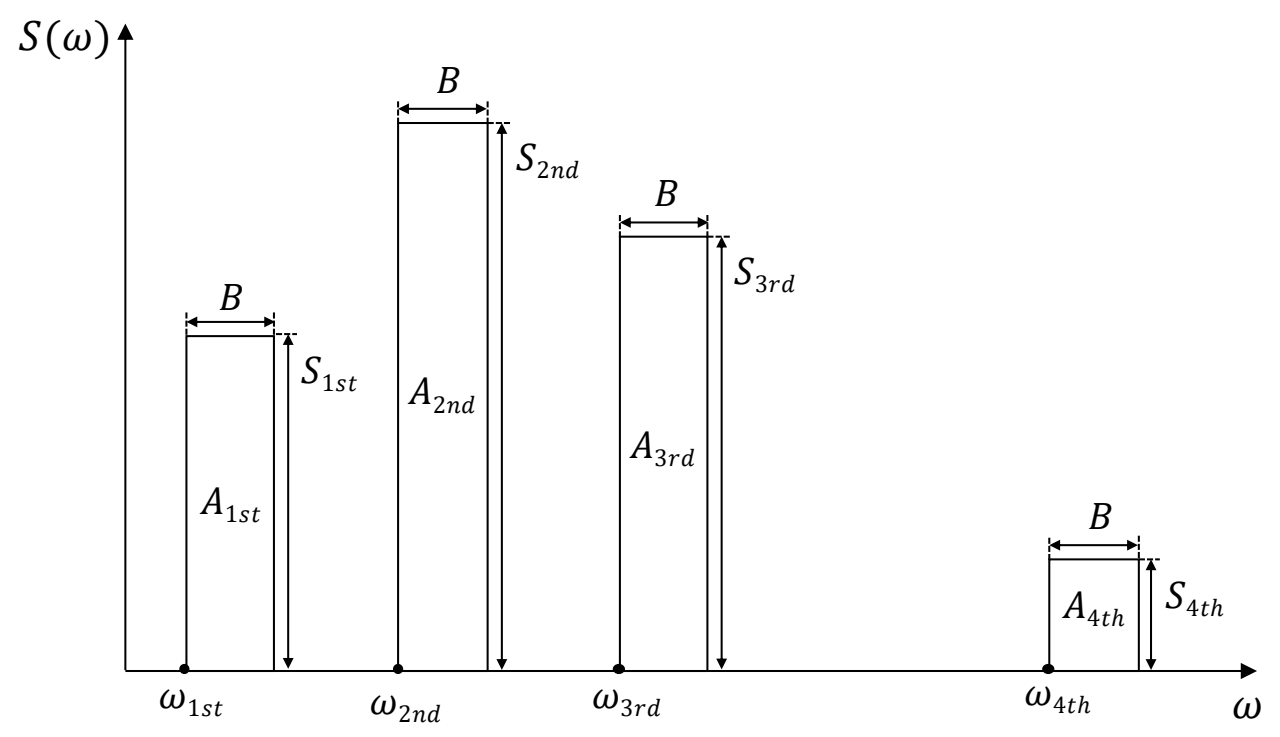

Fig. 2 Schematic of a quarter-modal spectrum

The $\omega_{1 s t}, \omega_{2 n d}, \omega_{3 r d}$, and $\omega_{4 t h}$ denote the starting frequency of each mode peak, and they were determined in consideration of the characteristic of each mode peak, respectively. The first mode peak is a low-frequency ship motion generated by a low-frequency drift force. Therefore, the $\omega_{1 s t}$ is conventionally in the range of $0.02 \mathrm{rad} / \mathrm{s} \sim 0.2 \mathrm{rad} / \mathrm{s}$ [20]. The second mode peak is caused by the wave-frequency ship motion and is determined by dominant wave frequency at the offshore platform's installation site. The third and fourth mode peaks are generated by VIV, and the diameter of the riser as well as the current velocity should be considered in order to identify the VIV frequency. The location of the third peak $\omega_{3 r d}$ can be determined, by substituting the diameter of the riser and the velocity of fluid into the shedding frequency equation (18) [21].

$$
f_{\text {shedding }}=\frac{2 \pi * U * S_{t}}{D}
$$

where $S_{t}$ is a dimensionless number of shedding characteristic and its conventional value during VIV occurrence is 0.2 . $U$ is velocity of fluid, and $D$ is diameter of the riser. Because the frequency of occurrence of the fourth mode peak, which is an in-line (IL), is usually twice the frequency of a cross-flow (CF) in the experimental research [22], $\omega_{4 t h}$ is twice of $\omega_{3 r d}$.

In order to generate ideal spectra, various peak locations were considered by reflecting the characteristics of each mode peak. Given that the frequency range of the first mode peak is relatively smaller than the frequency variation of all the other peaks, $\omega_{1 s t}$ was fixed to be 0.05 $\mathrm{rad} / \mathrm{s}$, which is a sufficiently low frequency region. As shown in equation (19), the variable $d$ was used to locate $\omega_{2 n d}$ at $25 \%, 50 \%$, and $75 \%$ between $\omega_{1 s t}$ and $\omega_{3 r d}$.

$$
\omega_{2 n d}=\omega_{1 s t}+\left(\omega_{3 r d}-\omega_{1 s t}\right) * d
$$

where $\omega_{3 r d}$ is located in the range of $2 \mathrm{rad} / \mathrm{s} \sim 12.8 \mathrm{rad} / \mathrm{s}$, and $\omega_{4 t h}$ is twice of $\omega_{3 r d}$. The maximum frequency was considered as $26.5 \mathrm{rad} / \mathrm{s}$. Table 1 . present the values of the applied spectra. 
Table 1 Location parameters of model developing quarter-modal spectra

\begin{tabular}{|c|c|}
\hline Parameters & Value \\
\hline$A_{T}$ & 5,000 \\
\hline$B[\mathrm{rad} / \mathrm{s}]$ & $0.2,0.4$ \\
\hline$d$ & $0.25,0.50,0.75$ \\
\hline$\omega_{1 s t}[\mathrm{rad} / \mathrm{s}]$ & 0.05 \\
\hline$\omega_{3 r d}[\mathrm{rad} / \mathrm{s}]$ & $2.0,7.4,12.8$ \\
\hline$\omega_{4 t h}[\mathrm{rad} / \mathrm{s}]$ & $\omega_{3 r d} \times 2.0$ \\
\hline Max freq. $[\mathrm{rad} / \mathrm{s}]$ & 26.5 \\
\hline
\end{tabular}

The width of each peak is expressed as $B$, and the total area $A_{T}$ of the spectrum is calculated by the sum of areas of all the mode peaks, as shown in equation (20),

$$
A_{T}=A_{1 s t}+A_{2 n d}+A_{3 r d}+A_{4 t h}
$$

Where $A_{1 s t}, A_{2 n d}, A_{3 r d}$, and $A_{4 t h}$ are the area for each mode peak. And two values of $0.2 \mathrm{rad} / \mathrm{s}$ and $0.4 \mathrm{rad} / \mathrm{s}$ were used for $B$.

Since the spectra used in this study are not real responses, the areas of spectrum $A_{T}$ was arbitrarily set to be 5,000. When deriving the fatigue damage models, magnitude of the area is not an important factor while the statistical properties of spectrum are matter.

The area of each mode peaks was calculated by equations (21) to (23).

$$
\begin{aligned}
& R_{1 s t}=\frac{A_{1 s t}}{A_{1 s t}} \\
& R_{2 n d}=\frac{A_{2 n d}}{A_{1 s t}} \\
& R_{3 r d}=\frac{A_{3 r d}}{A_{1 s t}}
\end{aligned}
$$

where $R_{1 s t}, R_{2 n d}, R_{3 r d}$, and $R_{4 t h}$ are variables expressing the ratios of area between each mode peak.

The $R_{1 s t}$ is always 1 by its definition. For the $R_{2 n d}$ and $R_{3 r d}$, the various random ratios are used in order to generate many kinds of spectra. For $R_{4 t h}$, the two values of $R_{3 r d}, 1 / 5$ and $1 / 20$, were used.

The spectral density for each mode peak were calculated by using the equations of quarter-modal spectral density, which are presented in equations (24) to (27) as below [23].

$$
S_{1 s t}=\frac{A_{1 s t}}{B}=\frac{A_{T} R_{1 s t}}{B\left(R_{1 s t}+R_{2 n d}+R_{3 r d}+R_{4 t h}\right)}
$$




$$
\begin{gathered}
S_{2 n d}=\frac{A_{2 n d}}{B}=\frac{A_{T} R_{2 n d}}{B\left(R_{1 s t}+R_{2 n d}+R_{3 r d}+R_{4 t h}\right)} \\
S_{3 r d}=\frac{A_{3 r d}}{B}=\frac{A_{T} R_{3 r d}}{B\left(R_{1 s t}+R_{2 n d}+R_{3 r d}+R_{4 t h}\right)} \\
S_{4 t h}=\frac{A_{4 t h}}{B}=\frac{A_{T} R_{4 t h}}{B\left(R_{1 s t}+R_{2 n d}+R_{3 r d}+R_{4 t h}\right)}
\end{gathered}
$$

where $S_{1 s t}, S_{2 n d}, S_{3 r d}$, and $S_{4 t h}$ are spectral density of each mode peak.

Table 2. present the variables and values of the applied spectra.

Table 2 Area ratios of model developing quarter-modal spectra

\begin{tabular}{|c|c|c|c|}
\hline \multicolumn{3}{|c|}{ Area ratios } \\
\hline \multirow{3}{*}{$R_{1 s t}$} & $R_{2 n d}$ & $R_{3 r d}$ & \multirow{2}{*}{$R_{4 t h}$} \\
\hline \multirow{4}{*}{1} & 0.04 & $0.04,1,2$ & \multirow{2}{*}{$R_{3 r d} / 5$} \\
\cline { 2 - 3 } & 0.02 & $0.02,0.5,1$ & \multirow{2}{*}{$R_{3 r d} / 20$} \\
\cline { 2 - 3 } & 0.5 & $0.02,0.5,1$ & \\
\cline { 2 - 3 } & 1 & $0.04,0.02,0.5,1,2,25,50$ & \\
\cline { 2 - 3 } & 2 & $0.04,1,2$ & \\
\cline { 2 - 3 } & 25 & $1,25,50$ & \\
\cline { 2 - 3 } & 50 & $1,25,50$ & \\
\hline
\end{tabular}

Finally, a total 900 spectra were considered for developing fatigue damage model. They are the combinations of the 9 locations of mode peaks, the 25 relative area ratios between mode peaks, the 2 widths of mode peaks, and the 2 area ratios of fourth mode peaks. Fig. 3 shows an example of a wide-band quarter-modal spectrum and its time series which are used for developing the model. 


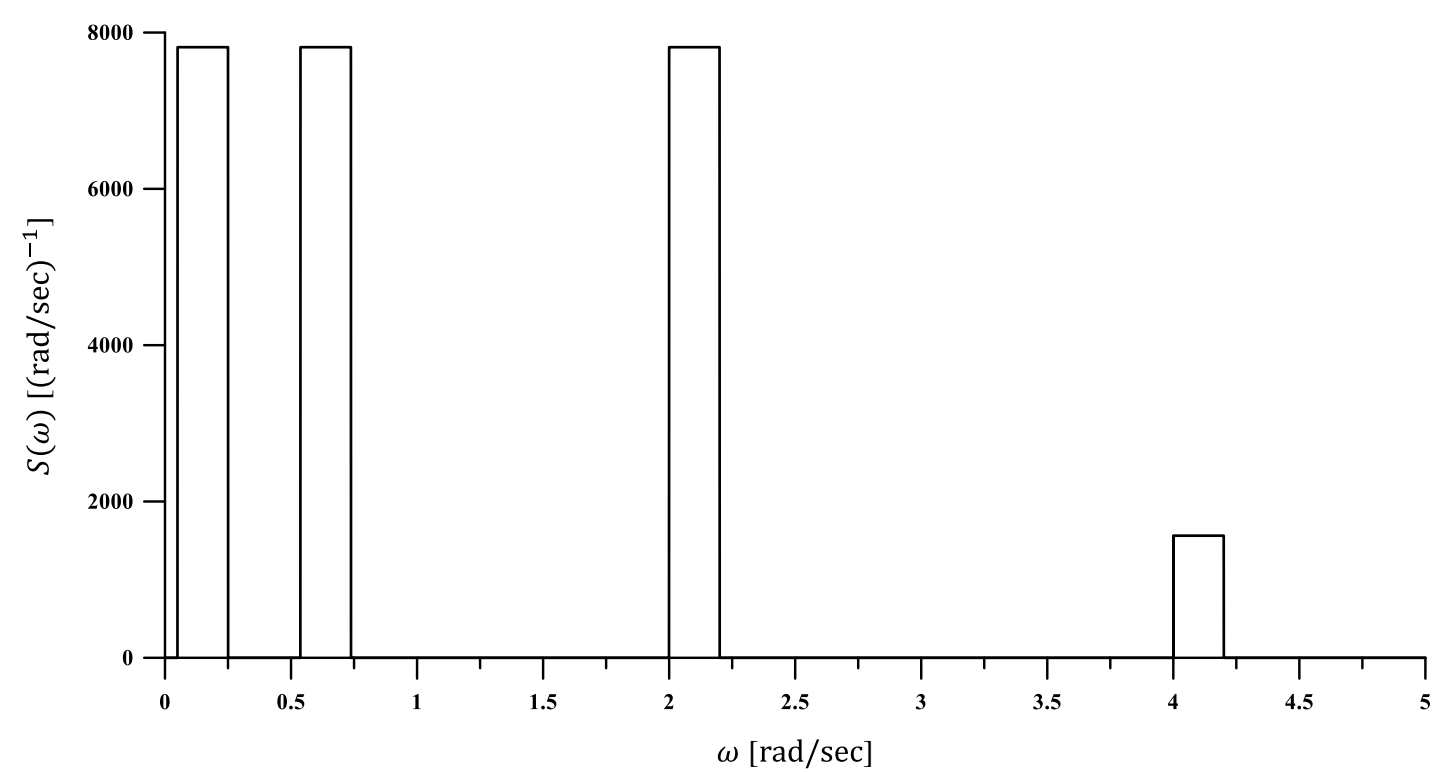

(a)

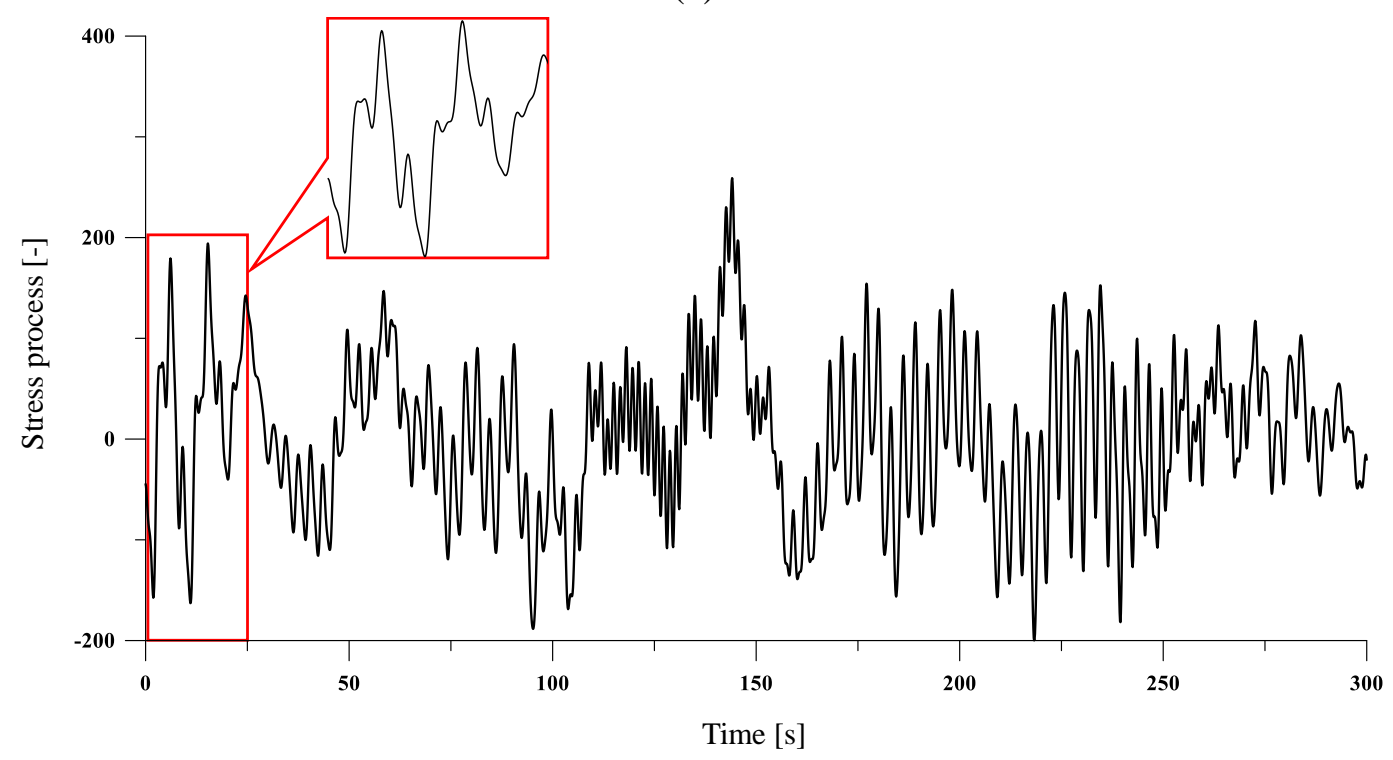

(b)

Fig. 3 Example of a quarter-modal spectrum (a) and time series(b)

\subsection{Numerical simulation}

\subsubsection{Calculation procedure of time domain fatigue damage}

The inverse Fourier transform (IFT) is used for the process of generating a time series from a spectrum in order to calculate the time domain fatigue damage of the spectrum as shown in the equations (28) and (29),

$$
X(t)=\sum_{i=1}^{n} \sqrt{2 S\left(\bar{\omega}_{i}\right) \Delta \omega} \cos \left(\bar{\omega}_{i} t+\theta_{i}\right)
$$




$$
\bar{\omega}_{i}=\frac{\omega_{i}+\omega_{i-1}}{2}
$$

where $n$ is the number of frequency components. It was determined by dividing a set maximum frequency by the size of the frequency increment. $S\left(\bar{\omega}_{i}\right)$ is the spectral density of the $i^{t h}$ frequency component, $\Delta \omega$ is the frequency increment, $\bar{\omega}_{i}$ is the $i^{\text {th }}$ average frequency, and $\theta_{i}$ is the random phase angle of the $i^{\text {th }}$ frequency component.

The $\Delta \omega$ was set to be $0.0005 \mathrm{rad} / \mathrm{s}$. Such an equally spaced value is small enough not to make a repetition occur during the numerical simulation in a time series generated from spectrum. The size of the time increment, $\Delta t$, should be set to be small enough to distinguish between peak and valley in a time series. The size of $\Delta t$ was calculated by equation (30),

$$
\Delta t=\frac{0.08 * 2 \pi}{\text { Max freq. }}
$$

where the ratio between the maximum frequency of spectrum suggested by a previous study [24] and the time increment was set to be 0.08 .

An ensemble of time series was generated for 3 hours from one spectrum by using random phases. The rainflow counting method was used to calculate fatigue damage of generated time series. The applied rainflow counting method was the 3-points algorithm proposed by ASTM [25].

\subsubsection{Deriving a new linear weighting factor}

To begin with, the values of $\alpha_{1}$ and $\alpha_{2}$ are calculated for every spectrum used for the model development. In order to derive the linear weighting factor, as shown in equation (31), $b_{\text {sim }}$ determined by the time domain and frequency domain methods should be calculated.

$$
b_{\text {sim }}=\frac{D_{R F C}-E\left(D_{R C}\right)}{E\left(D_{N B}\right)-E\left(D_{R C}\right)}
$$

To calculate the $D_{R F C}$, as described in previous chapter, the 3-points algorithm used, and the slope of S-N curve $k$ and material constant $C$ which are the main parameters of calculation were set to 3 and 1 . The value of $k$ is mainly suggested by DNV [20] for riser design. The material constant $C$ has no effects on the weighting factor as equation (31). And the trend of linear weighting factor can be more easily found since the deviation of fatigue damage is small when $k$ value is 3 rather than 5 .

As the spectra were derived by various variables, the values of $\alpha_{1}, \alpha_{2}$, and $b_{\text {sim }}$ are different for each spectrum. In order to identify the trend lines of $\alpha_{1}, \alpha_{2}$, and $b_{\text {sim }}$, the calculated values of $\alpha_{2}$ were modified to five values of $0.15,0.325,0.500,0.675$, and 0.850 . When modifying the values of $\alpha_{2}$, the ratios of areas were randomly adjusted in order to determine various pairs of $\alpha_{1}$ and $\alpha_{2}$. The 900 spectra are classified into 68, 61, 221, 382, and 168 with respect to the designated values of $\alpha_{2}$. 


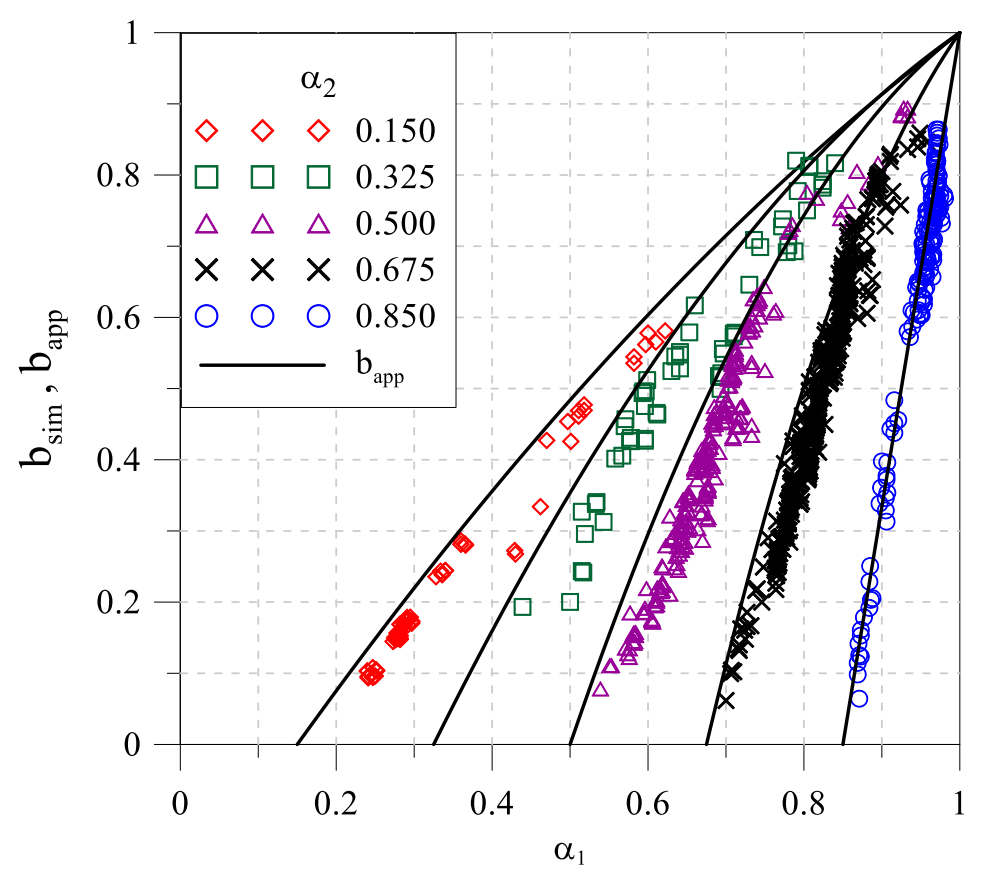

Fig. 4 Comparison result between $b_{\text {sim }}$ and $b_{a p p}(k=3)$

In Fig. 4 , the values of $b_{\text {sim }}$ calculated in terms of the modified spectra are presented in relation to $\alpha_{1}$ and $\alpha_{2}$. Moreover, $b_{a p p}$ is presented to compare the existing model with the result of the numerical simulation. As a result, $b_{a p p}$ has a different trend from that of $b_{s i m}$ of a wide-band quarter-modal spectrum, and the error of trend becomes larger at small $\alpha_{1}$. This represents that the wide-band characteristic of the spectrum is clearer at small $\alpha_{1}$. This is because the spectra used to develop the model are different. When the previous model was developed, five spectral shapes are defined as wideband-spectra, and their spectral parameters were investigated [7]. In this study, however, the new quarter model spectrum that does not belong to five types is investigated. That's the reason why the new weighting factor is different with the previous weighting factor.

A new linear weighting factor for $\alpha_{1}$ and $\alpha_{2}$ was derived in order to reduce the error in the existing model, and this new factor was defined as $s$. The method of deriving the new weighting factor $\mathrm{s}$ was the least square method, which makes a curve where the sum of squares of residuals for each data is minimum, and various types of objective functions suitable for $b_{\text {sim }}$ were determined. The validity of the selected objective functions was examined by using a coefficient of determination, which is a measure of the adequacy of $b_{s i m}$ and the objective functions. The coefficient of determination is the square of the objective functions and a coefficient of correlation, $r$, which expresses the degree of correlation between $b_{\text {sim }}$ and the objective functions. It is expressed in equation (32).

$$
r^{2}=\frac{\sum_{i=1}^{900}\left(b_{s i m, i}-\bar{b}_{s i m}\right)^{2}-\sum_{i=1}^{900}\left(b_{s i m, i}-s\left(\alpha_{1}, \alpha_{2}\right)\right)^{2}}{\sum_{i=1}^{900}\left(b_{s i m, i}-\bar{b}_{s i m}\right)^{2}}
$$

Here, $\bar{b}_{\text {sim }}$ is defined by the following equation (33). 


$$
\bar{b}_{s i m}=\frac{1}{900} \sum_{i=1}^{900} b_{\text {sim }, i}
$$

Four objective functions are used to find the optimal objective function with the coefficient of determination close to 1 . The applied objective functions are full cubic, rational, full quadratic, and simplified cubic functions. For each objective function, s was derived by applying the least square method, as shown in equations (34) to (37).

$$
\begin{aligned}
s_{\text {Full cubic }}= & -0.06-0.26 \alpha_{1}+1.72 \alpha_{2}+3.77 \alpha_{1}^{2}-5.19 \alpha_{2}^{2}-1.71 \alpha_{1}^{3} \\
& -8.73 \alpha_{2}^{3}-3.64 \alpha_{1} \alpha_{2}-4.17 \alpha_{1}^{2} \alpha_{2}+18.68 \alpha_{1} \alpha_{2}^{2} \\
s_{\text {Full quadratic }}= & 0.07+2.01 \alpha_{1}-3.53 \alpha_{2}-2.63 \alpha_{1}^{2}-4.53 \alpha_{2}^{2}+9.06 \alpha_{1} \alpha_{2} \\
s_{\text {Rational }}= & \frac{-0.089+1.55 \alpha_{1}-1.47 \alpha_{2}}{1+0.35 \alpha_{1}-1.31 \alpha_{2}} \\
s_{\text {Simplified cubic }}= & -0.59+4.88 \alpha_{1}-0.76 \alpha_{2}-8.22 \alpha_{1}^{2}+0.54 \alpha_{2}^{2}+5.93 \alpha_{1}^{3}-1.33 \alpha_{2}^{3}
\end{aligned}
$$

Table 3. presents the coefficients of determination and correlation for each objective function. Fig. 5 to Fig. 8 compares each model and the $b_{\text {sim }}$ to identify adequacy.

Table 3 Correlation coefficient and determination of coefficient for each model

\begin{tabular}{|c|c|c|}
\hline Model & $r$ & $r^{2}$ \\
\hline Full cubic & 0.981 & 0.962 \\
\hline Full quadratic & 0.970 & 0.941 \\
\hline Rational & 0.978 & 0.957 \\
\hline Simplified cubic & 0.955 & 0.911 \\
\hline
\end{tabular}




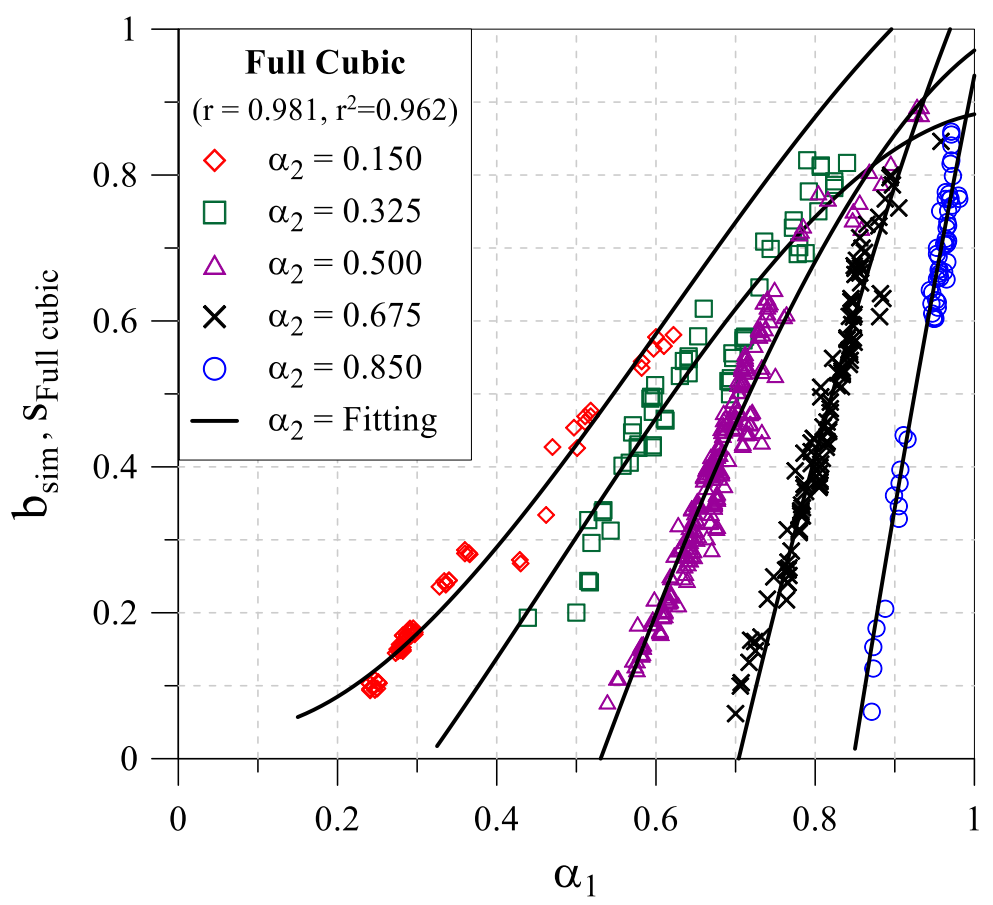

Fig. 5 Least square method results for each object function ( $k=3$, Full cubic)

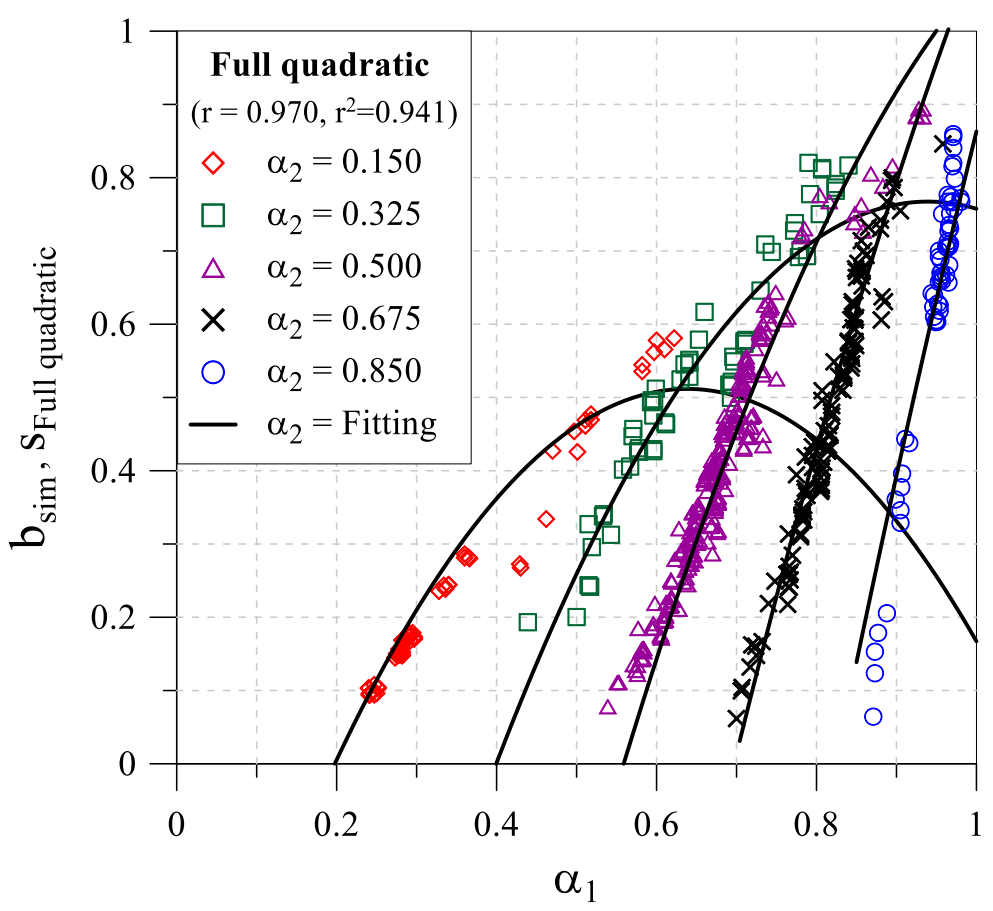

Fig. 6 Least square method results for each object function ( $k=3$, Full quadratic) 


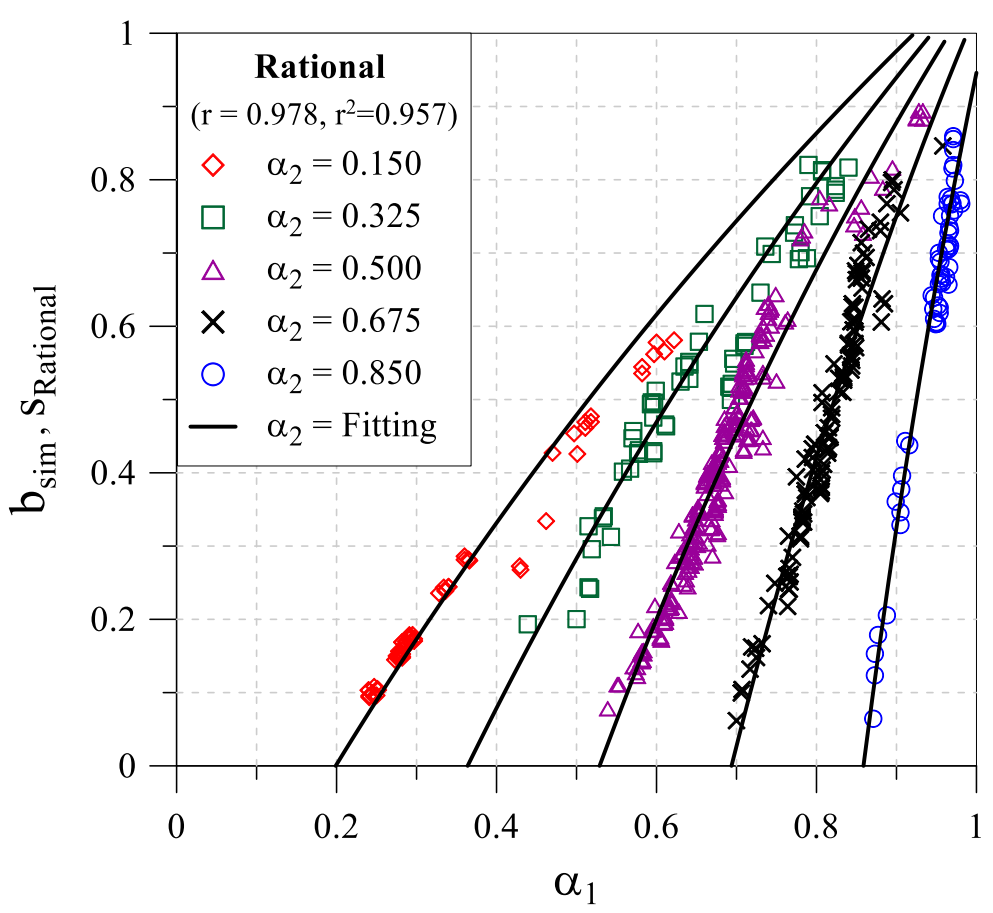

Fig. 7 Least square method results for each object function $(k=3$, Rational)

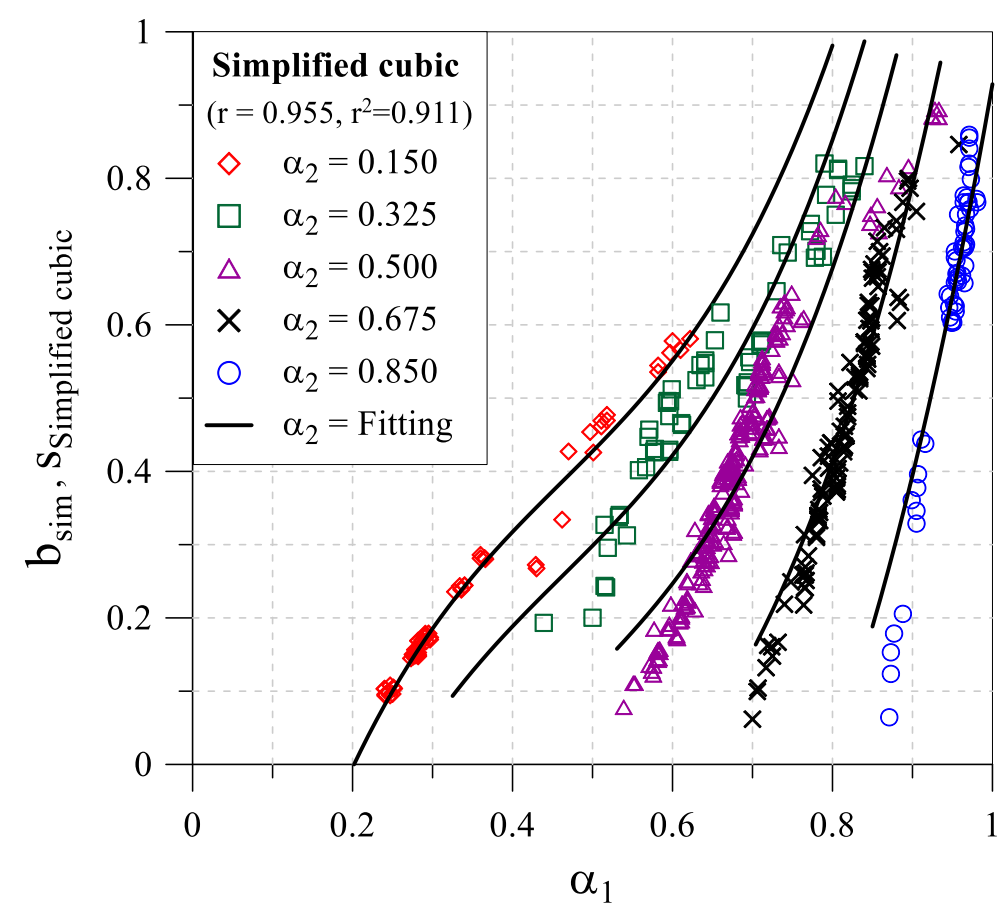

Fig. 8 Least square method results for each object function ( $k=3$, Simplified cubic)

Although the full cubic function had a high coefficient of determination, it shows large deviation of trend for $\alpha_{2}$. On the other hand, the rational function was found to be the most appropriate function, and the derived coefficient $s$ is as shown in equation (38)

$$
s=\frac{-0.089+1.55 \alpha_{1}-1.47 \alpha_{2}}{1+0.35 \alpha_{1}-1.31 \alpha_{2}}
$$

A new fatigue damage model for quarter-modal spectrum, using $s$, is as shown in equation (39). 


$$
E\left(D_{R F C}\right)=s E\left(D_{N B}\right)+(1-s) E\left(D_{R C}\right)=\left[s+(1-s) \alpha_{2}^{(k-1)}\right] E\left(D_{N B}\right)
$$

The new equation must satisfy two boundary conditions. The first boundary condition is that maximum $b_{a p p}$ is 1 according to the definition of linear weighting factor. The second is that if $\alpha_{1}$ and $\alpha_{2}$ have the same value, the $b_{a p p}$ is zero. However, both conditions can be met only when the spectrum is a delta function. So, to satisfy two boundary conditions, additional conditions expressed in equations (40) and (41) were added.

$$
\begin{aligned}
& s=0 \text { when } s<0 \\
& s=1 \text { when } s>1
\end{aligned}
$$

\section{Validation of the developed model}

\subsection{Spectra for validation}

In order to validate the developed fatigue damage model, the combinations of 9 locations of mode peaks and the 9 relative area ratios of mode peaks, totally 81 spectra, were considered refer to previous researches [23]. And for the $R_{2 n d}$ and $R_{3 r d}$, the values proposed by Park et al. [26] using a tri-modal spectrum were used in order to consider various number of cases. The value of $R_{4 t h}$ was set to be $1 / 10$ of $R_{3 r d}$ in order to be small enough compared to $R_{3 r d}$. As in chapter 3 the spectral density of each mode peak was calculated by using the equations (20) to (27). The $A_{T}$ was set to 5,000 , and $B$ was set to $0.2 \mathrm{rad} / \mathrm{s}$, to express a narrow-band peak. Table 4. present the values of the applied parameters.

Table 4 Parameters of validation spectra

\begin{tabular}{|c|c|}
\hline Parameters & Parametric value \\
\hline$A_{T}$ & 5,000 \\
\hline$B[\mathrm{rad} / \mathrm{s}]$ & 0.2 \\
\hline$\omega_{1 s t}[\mathrm{rad} / \mathrm{s}]$ & 0.05 \\
\hline$\omega_{3 r d}[\mathrm{rad} / \mathrm{s}]$ & $2.0,7.25,12.5$ \\
\hline$\omega_{4 t h}[\mathrm{rad} / \mathrm{s}]$ & $\omega_{3 r d} \times 2.0$ \\
\hline$R_{1 s t}$ & 1 \\
\hline$R_{2 n d}$ & $10^{-0.8}, 10^{0}, 10^{0.8}$ \\
\hline$R_{3 r d}$ & $10^{-0.8}, 10^{0}, 10^{0.8}$ \\
\hline$R_{4 t h}$ & $R_{3 r d} / 10$ \\
\hline Max freq. $[\mathrm{rad} / \mathrm{s}]$ & 25.2 \\
\hline$\Delta \omega[\mathrm{rad} / \mathrm{s}]$ & 0.0005 \\
\hline$\Delta t[\mathrm{~s}]$ & 0.02 \\
\hline
\end{tabular}




\subsection{Validation result}

To evaluate the suitability of the new fatigue analysis model for the spectrum of validation, we calculated the expected value of the fatigue damage by using the Benasciutti and Tovo model, the Dirlik model, and the new model. The fatigue damage calculated for the spectrum of validation by the time domain fatigue analysis method was set to be the reference fatigue damage, and the expected value calculated by each fatigue damage model was divided by the reference damage. As the result becomes closer to 1 , it can be said that the reliability of the applied model increases. The calculated results are expressed by the Vanmarcke's bandwidth parameter $q$ [27], as shown in equation (42).

$$
q=\sqrt{1-\frac{m_{1}^{2}}{m_{0} m_{2}}}
$$

The $q$ is a variable that quantitatively expresses the wide-band and narrow-band characteristics of spectra, and it is close to zero for a narrow-band spectrum, while it is close to one for a wide-band spectrum.

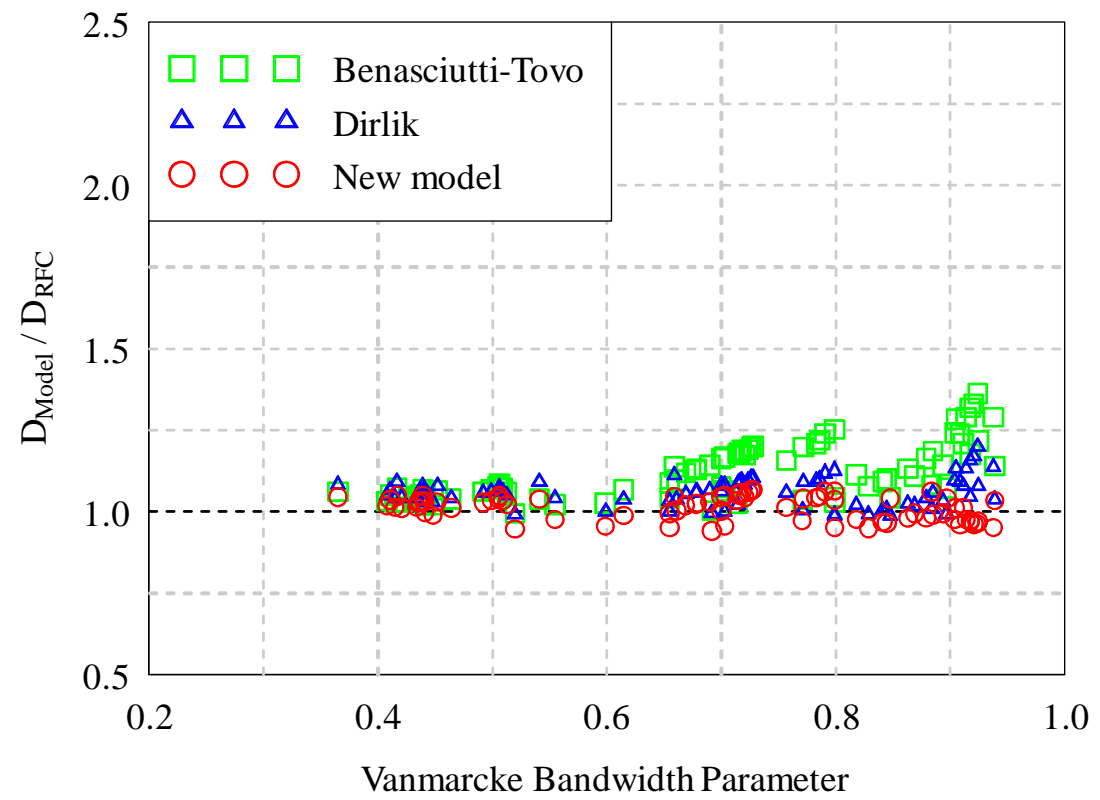

Fig. 9 Validation result of new model $(k=3)$ 


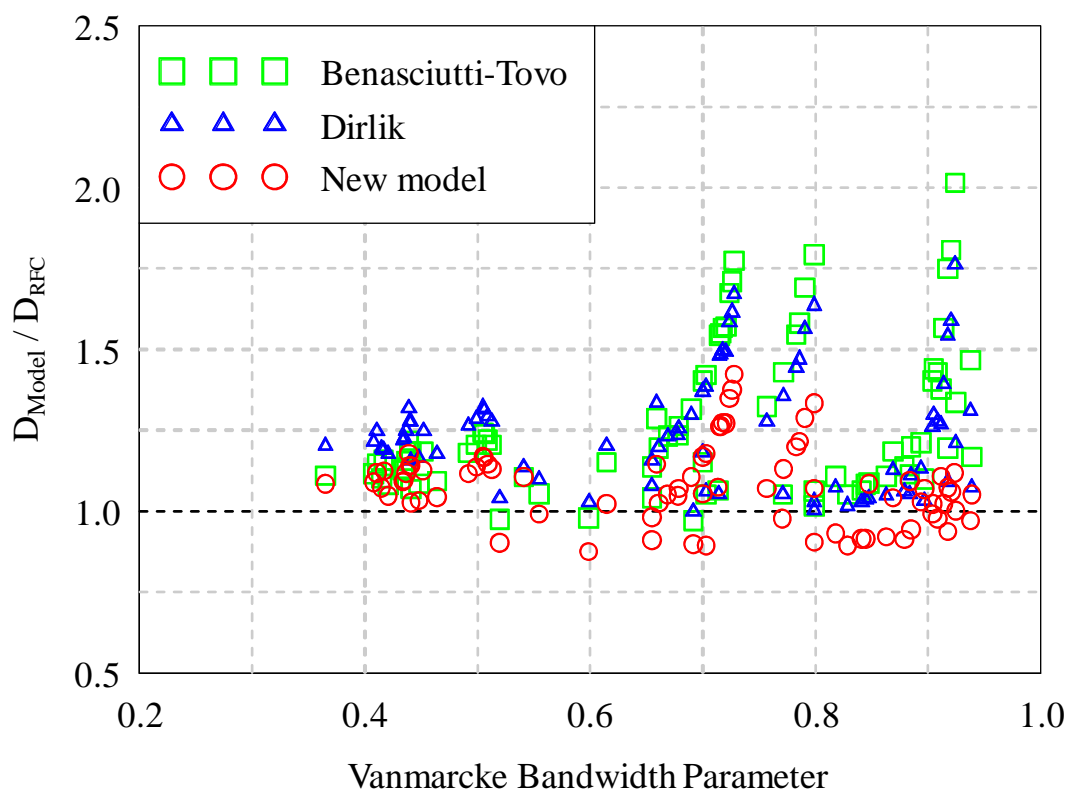

Fig. 10 Validation result of new model $(k=5)$

The fatigue damages were calculated for two cases kinds of $k$ were 3 and 5. Fig. 9 and 10 shows the result of the calculation. When the $k$ was 3 , which is the case (a), the Benasciutti and Tovo model generally produced a larger deviation from 1 than the other two models. In particular, the deviation was much larger at the region where the $q$ was large. The Dirlik model also had a larger deviation than the new model at this region. On the other hand, when the $k$ was 5 , the Benasciutti and Tovo model and the Dirlik model had larger deviations than the new model. The new model generated a larger deviation than when the $k$ was 3 , but its deviation was smaller than those of the other two models. As a result, the newly developed model was more stable than other two models for evaluating the fatigue damage of a wideband quarter-modal spectrum.

\section{Conclusion}

In this study, a new fatigue damage model for quarter-modal spectrum is proposed. The riser stress response was selected for quarter-modal spectrum including the low- and wavefrequency ship motions, and CF and IL of the VIV phenomenon. For developing the new fatigue damage model, the Benasciutti and Tovo model was used. 900 wide-band quartermodal spectra for model development were defined and the linear weighting factor was calculated for each spectrum. The results are validated for two bandwidth variables so that the relation between the bandwidth variable and the linear weighting factor.

In order to validate developed fatigue damage model, the comparison between existing wide-band fatigue model and developed model was presented in both frequency domain and the time domain. The results show that the developed model produces adequate evaluation of the fatigue damage, while the existing wide-band fatigue damage models presents excessive fatigue damage.

\section{REFERENCES}

[1] Matsuishi, M., Endo, T. (1968). Fatigue of metals subjected to varying stress. Japan Society of Mechanical Engineers, Fukuoka, Japan, 37-40. 
[2] Miner, M.A. (1945). Cumulative damage in fatigue. Journal of Applied Mechanics, 12, 159-164.

[3] Dirlik, T. (1985). Application of computers in fatigue. Ph.D Thesis, University of Warwick.

[4] Zhao, W., Baker, M.J. (1992). On the probability density function of rainflow stress range for stationary Gaussian processes. International Journal of Fatigue 14(2), 121-135. https://doi.org/10.1016/0142$\underline{1123(92) 90088-\mathrm{T}}$

[5] Bendat, J.S. (1964). Probability functions for random responses: prediction for peaks, fatigue damage, and catastrophic failures. National Aeronautics and Space Administration, 33.

[6] Wirsching, P.H., Light, M.C. (1980). Fatigue under wideband random stresses. Journal of the Structural Division, ASCE (American Society of Civil Engineers), 106(7), 1593-1607.

[7] Benasciutti, D., Tovo, R. (2005). Spectral methods for life time prediction under wide-band stationary random processes. International Journal of Fatigue, 27(8), 867-877. https://doi.org/10.1016/j.ijfatigue.2004.10.007

[8] Fu, T.T., Cebon, D. (2000). Predicting fatigue lives for bi-modal stress spectral densities. International Journal of Fatigue, 22(1), 11-21. https://doi.org/10.1016/S0142-1123(99)00113-9

[9] Sakai, S., Okamura, H. (1995). On the distribution of rainflow range for Gaussian random processes with bimodal PSD. JSME International Journal. Ser. A, Mechanics and Material Engineering, 38(4), 440-445. https://doi.org/10.1299/jsmea1993.38.4 440

[10] Jiao, G., Moan, T. (1990). Probabilistic analysis of fatigue due to Gaussian load processes. Probabilistic Engineering Mechanics, 5(2), 76-83. https://doi.org/10.1016/0266-8920(90)90010-H

[11] Gao, Z., Moan, T. (2008). Frequency-domain fatigue analysis of wide-band stationary Gaussian processes using a trimodal spectral formulation. International journal of fatigue, 30(10), 1944-1955. ttps://doi.org/10.1016/j.ijfatigue.2008.01.008

[12] Park, J.B., Choung, J.M., Kim, K.S. (2014). A new fatigue prediction model for marine structures subject to wideband stress process. Ocean Engineering, 76, 144-151. https://doi.org/10.1016/j.oceaneng.2013.11.002

[13] DNV (2005). Recommended practice DNV-RP-F204 riser fatigue. Det Norske Veritas (DNV), Norway

[14] Zhao, S., Chen, C., Fan, T., Jiang, Y., Shen, Y. (2019). Fatigue assessment of crack growth based on failure assessment diagrams for a semi-submersible platform. Brodogradnja, 70(1). 11-24 https://doi.org/10.21278/brod70102

[15] Hejazi, R., Grime, A., Randolph, M., Efthymiou, M. (2018). An ANN-based framework for rapid spectral fatigue analysis of steel catenary risers. Offshore Technology Conference Asia 2018, Kuala Lumpur, Malaysia, March. https://doi.org/10.4043/28397-MS

[16] Wong, E.W.C., Kim, D.K. (2018). A simplified method to predict fatigue damage of TTR subjected to short-term VIV using artificial neural network. Advances in Engineering Software, 126, 100-109. https://doi.org/10.1016/j.advengsoft.2018.09.011

[17] Ulveseter, J.V., Thorsen, M.J., Sævik, S., Larsen, C.M. (2018) Time domain simulation of riser VIV in current and irregular waves. Marine Structures, 60, 241-260 https://doi.org/10.1016/j.marstruc.2018.04.001

[18] Benasciutti, D., Tovo, R (2006) Comparison of spectral methods for fatigue analysis in broad-band Gaussian random processes. Probabilistic Engineering Mechanics, 21(4), 287-299. https://doi.org/10.1016/j.probengmech.2005.10.003

[19] Madsen, H.O., Krenk, S., Lind, N.C. (1986). Methods of structural safety. Prentice-Hall, Englewood Cliffs, N.J.

[20] DNV (2010). DNV-RP-C205 Environmental conditions and environmental loads. Det Norske Veritas, Norway.

[21] Robert, D.B. (2001). Flow-Induced Vibration. Reprint Edition, Malabar, Florida, Krieger Publishing.

[22] Kim, S.W., Lee, S.J., Park, C.Y., Kang, D.H. (2016). An experimental study of a circular cylinder's two-degree-of-freedom motion induced by vortex. International Journal of Naval Architecture and Ocean Engineering, 8(4), 330-343. https://doi.org/10.1016/j.ijnaoe.2016.05.001

[23] Kim, S.W., Lee, S.J., Choi, S.M. (2016). A study on the riser fatigue analysis using a quarter-modal spectrum. Journal of the Society of Naval Architects of Korea, 53(6). https://doi.org/10.3744/SNAK.2016.53.6.514

[24] Park, J.B., Kim, K.S., Choung, J.M., Yoo, C.H., Ha, Y.S. (2011). Data acquisition of time series from stationary ergodic random process spectrums. Journal of Ocean Engineering and Technology 25, 120126. https://doi.org/10.5574/KSOE.2011.25.2.120 
[25] ASTM International. (1990). Standard Practices for Cycle Counting in Fatigue Analysis. American Society for Testing and Materials, E1049-85.

[26] Park, J.B., Chang, Y.S. (2015). Fatigue damage model comparison with formulated tri-modal spectrum loadings under stationary Gaussian random processes. Ocean Engineering 105, 72-82. https://doi.org/10.1016/j.oceaneng.2015.05.039

[27] Vanmarcke, E.H. (1972). Properties of spectral moments with applications to random vibration. Journal of the Engineering Mechanics Division, 98(2), 425-446.

Submitted: $\quad$ 05.09.2018. $\quad$ Sang Woo Kim, sangwookim.1201@gmail.com

Accepted: $\quad$ 06.02.2020. $\quad \begin{aligned} & \text { Seung Jae Lee, slee@ kmou.ac.kr } \\ & \text { Division of Naval Architecture and Ocean Systems Engineering, }\end{aligned}$ Korea Maritime and Ocean University, Busan, Republic of Korea 